\title{
In Situ TEM Observation of Solid Electrolyte Interface Using High Speed Direct Electron Camera
}

\author{
Liang Jin*, Benjamin E. Bammes, Dong-Hua Chen, Michael S. Spilman and Robert B. Bilhorn Direct \\ Electron, LP, 13240 Evening Creek Drive S., Suite 311, San Diego, CA 92128 USA
}

In Situ Transmission electron microscopy (TEM) is a powerful technique for visualizing dynamic processes at nanometer or Angstrom resolution. It is widely used in studying catalysis, batteries, material transformations, nanoparticle growth, and biological processes. Increasingly, existing TEM camera performance is not able to keep up with the demanding requirements of the cutting-edge in-situ experiments, where large field of view, high signal-to-noise ratio (SNR) and high temporal resolution are all important factors.

In recent years, direct electron cameras [1] have become mainstream, and commercially available from companies such as Direct Electron, LP. Newly released acquisition software allows the use of their DE12 camera for in-situ TEM, with unlimited continuous streaming capability at 12-megapixels resolution 4096x3072@40 fps or even higher at 1000fps with 0.5-megapixels (see TABLE 1). With direct electron detection, the camera's detective quantum efficiency (DQE) well exceeds traditional scintillator based fiber-optic coupled CCD cameras. As measured by collaborators at Brandeis University, the DE-12 camera has a DQE of 0.4 at $1 / 2$ Nyquist frequency [2], more than three times the performance of the CCD camera. The benefits of direct detection are clearly visible in the metal edge images (FIG 1), which indicates much improved modulation transfer function (MTF) in the DE-12.

The unique high speed readout in the DE-12 camera allows parallel readout during the exposure and thus creating a continuous movie with virtually $100 \%$ duty cycle [3], a dramatic improvement over CCD camera whose readout time becomes a significant burden on duty cycle when operated at high frame rate.

The DE-12 camera was used recently in the direct observation of electrode-electrolyte interfaces in LiPF6/EC/DEC electrolyte for lithium ion batteries at our collaborator's lab at Lawrence Berkeley National Lab [4]. FIG 2 shows the Soliod Electrolyte Interface (SEI) formation bserved in situ. SEI is critical to the cycle ability in lithium ion batteries because it is formed in the process of the reduction of the solvent in the electrolyte. Previous studies of the SEI were mostly performed by ex situ techniques, and have many limitations. With in situ liquid cell TEM, it allowed imaging of the chemical reactions in real time using cyclic voltammetry. The separation of the SEI film from the Au electrode was observed, and can be attributed to gas produced during the active reaction [5].

In addition to liquid cell TEM, there are many in situ experiments that will benefit from the advancement of direct electron cameras. Further experiments in nano-mechanical tensile experiments, in situ Enviromental TEM (ETEM) and stroboscopic Dynamic TEM (DTEM) will be conducted.

\section{References}

[1] A.C. Milazzo, P. Leblanc, F. Duttweiler, L. Jin, et al., Ultramicroscopy, 104-2, 152 (2005).

[2] R.S. Ruskin, Z. Yu, N. Grigorieff, Journal of structural biology, 184.3, 385 (2013).

[3] L. Jin, A.C. Milazzo, et al., Journal of structural biology, 161.3, 352 (2008).

[4] Z. Zeng, W.I. Liang, H.G. Liao, et al., Nano letters, accepted (2014). 
[5] J. Christensen, et al., J. Electrochem. Soc., 151 (2004).

TABLE 1: Frame rates from example operation configurations of DE-12 DDD camera. The camera allows fully custom array size to suit any specific experimental needs.

\begin{tabular}{|l|l|c|c|}
\hline \multicolumn{1}{|c|}{ Configurations } & Image Size & Frame Rate & Effective Pixel Size \\
\hline Unbinned, 12 Megapixels & $4096 \times 3072$ & $40 \mathrm{fps}$ & $6 \mu \mathrm{m}$ \\
\hline Unbinned, 4 Megapixels & $2048 \times 2048$ & $108 \mathrm{fps}$ & $6 \mu \mathrm{m}$ \\
\hline Unbinned, 1 Megapixels & $1536 \times 512$ & $560 \mathrm{fps}$ & $6 \mu \mathrm{m}$ \\
\hline Unbinned, 0.5 Megapixels & $1792 \times 256$ & $1000 \mathrm{fps}$ & $6 \mu \mathrm{m}$ \\
\hline Bin 2, 3 Megapixels & $2048 \times 1536$ & $70 \mathrm{fps}$ & $12 \mu \mathrm{m}$ \\
\hline Bin 2, 1 Megapixels & $2048 \times 512$ & $214 \mathrm{fps}$ & $12 \mu \mathrm{m}$ \\
\hline
\end{tabular}

FIG 1. Metal edge shadow images acquired on (a) $15 \mu \mathrm{m}$ pixel size scintillator-based fiber optic coupled CCD; and (b) $6 \mu \mathrm{m}$ pixel Direct Electron DE-12 camera. The DE-12 shows a much
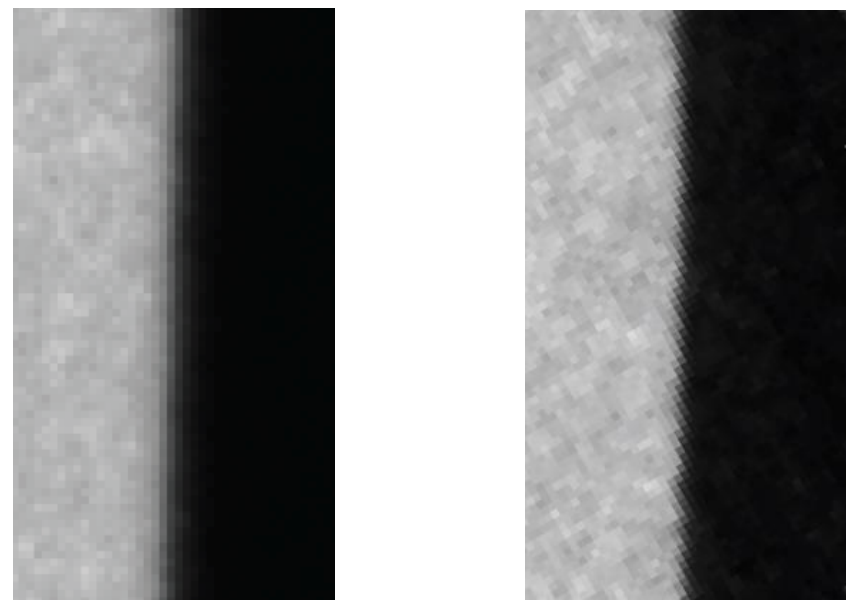

FIG 2. Single frame from the in situ movie of SEI film showing gas bubble emerged between the gold electrode and SEI film. The movie was captured at $75 \mathrm{fps}$ during cyclic voltammetry in the voltage range of 4 to $0 \mathrm{~V}$ at scan rate of $0.1 \mathrm{~V} / \mathrm{sec}$.

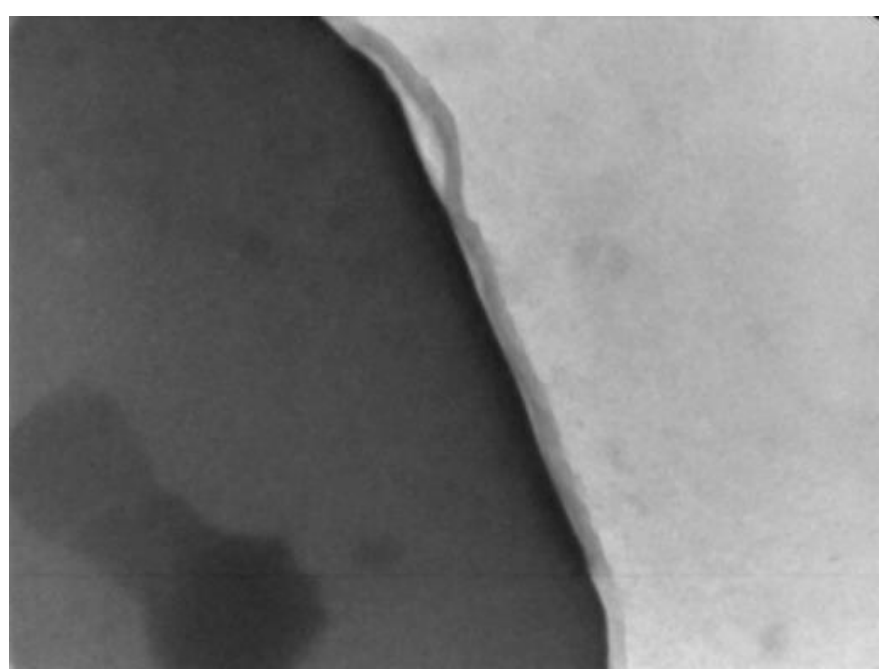

